\title{
Impact of Sedentary Lifestyle on Teenagers 13-25 Years in Urban Households
}

\author{
Pranav Dhawan* \\ Special Education Teacher at eduVelocity Global, Mumbai, India
}

\begin{abstract}
Through this paper the author aims to provide a high-level overview of the root cause of the issue of sedentary life. With the onset of the modern age came the onset of a new category of diseases, the diseases caused due to a sedentary lifestyle. With a fall of $30 \%$ since 1960 in jobs requiring physical activity, $80 \%$ of jobs today are sedentary or require only light activity. According to WHO, 60-85\% of the population does not engage in enough activity keeping this thought in mind the author through this paper aims to shows that the rate of diseases like obesity, cardiovascular diseases and diabetes type-2 has increased substantially since the 1950s, which may be attributed to the increased availability, affordability and range of different machines for different purposes which has made life easier and at the same time brought human energy expenditure to a relative stand still.
\end{abstract}

Keywords: Sedentary; Diabetes; Obesity; Cardiovascular diseases; Headaches

\section{INTRODUCTION}

Diabetes: A disease in which blood glucose, or blood sugar, levels are too high. Insulin is a hormone that helps the glucose gets into your cells to give them energy. With type-1 diabetes, your body does not make insulin. With type-2 diabetes, the more common type, your body does not make or use insulin well. Without enough insulin, the glucose stays in your blood. You can also have prediabetes. Over time, having too much glucose in your blood can cause serious problems. It can damage eyes, kidneys, and nerves. Pregnant women can also get diabetes, called gestational diabetes. Blood tests can show if you have diabetes. One type of test, the A1C, can also check on how you are managing your diabetes. Exercise, weight control and sticking to your meal plan can help control your diabetes [1].

According to the American Diabetes Association, in 2015, 30.3 million Americans, or $9.4 \%$ of the population, had diabetes. 1.5 million Americans are diagnosed with diabetes every year. Diabetes remains the 7 th leading cause of death in the United States in 2015 , with 79,535 death certificates listing it as the underlying cause of death, and a total of 252,806 death certificates listing diabetes as an underlying or contributing cause of death [2]. \$327 billion: Total costs of diagnosed diabetes in the United States alone in 2017 (Figures 1 and 2).

Obesity: Obesity is a medical condition in which excess body fat has accumulated to an extent that it may have a negative effect on health. People are generally considered obese when their body mass index (BMI), a measurement obtained by dividing a person's weight by the square of the person's height, is over $30 \mathrm{~kg} / \mathrm{m}^{2}$; the range $25-30 \mathrm{~kg} / \mathrm{m}^{2}$ is defined as Overweight [3].

Some East Asian countries use lower values. Obesity increases the likelihood of various diseases and conditions, particularly cardiovascular diseases, type-2 diabetes, obstructive sleep apnea, certain types of cancer, osteoarthritis, and depression. Obesity is most commonly caused by a combination of excessive food intake, lack of physical activity, and genetic susceptibility. A few cases are caused primarily by genes, endocrine disorders, medications, or mental disorder. The view that obese people eat little yet gain weight due to a slow metabolism is not medically

Correspondence to: Pranav Dhawan, Special Education Teacher at eduVelocity Global, Mumbai, India, Tel: +9815267009; E-mail: pranav.eduvelocity@gmail.com

Received: November 29, 2019; Accepted: January 23, 2020; Published: January 31, 2020

Citation: Dhawan P (2020) Impact of Sedentary Lifestyle on Teenagers 13-25 Years in Urban Households 8: 247. doi: 10.35248/23754273.20.8.247

Copyright: (C) 2020 Dhawan P. This is an open-access article distributed under the terms of the Creative Commons Attribution License, which permits unrestricted use, distribution, and reproduction in any medium, provided the original author and source are credited. 
supported $[4,5]$.

On an average, obese people have greater energy expenditure than their normal counterparts due to the energy required to maintain an increased body mass. Worldwide obesity has nearly tripled since 1975 . In 2016, more than 1.9 billion adults, 18 years and older, were overweight. Of these over 650 million were obese [6]. Most of the world's population lives in countries where overweight and obesity kills more people than underweight (Figure 3).

Cardiovascular Diseases: Cardiovascular disease (CVD) is a class of diseases that involve the heart or blood vessels. CVD includes coronary artery diseases (CAD) such as angina and myocardial infarction (commonly known as a heart attack).

The most common cardiovascular disease associated with sedentary life is coronary artery disease. Coronary artery disease develops when the major blood vessels that supply your heart with blood, oxygen and nutrients (coronary arteries) become damaged or diseased. Cholesterol-containing deposits (plaque) in your arteries and inflammation are usually to blame for coronary artery disease [7].

When plaque builds up, it narrows your coronary arteries, decreasing blood flow to your heart. Eventually, the decreased blood flow may cause chest pain (angina), shortness of breath, or other coronary artery disease signs and symptoms. A complete blockage can cause a heart attack [8].

Because coronary artery disease often develops over decades, you might not notice a problem until you have a significant blockage or a heart attack. But there's plenty you can do to prevent and treat coronary artery disease. A healthy lifestyle can make a big impact. 17.9 million People die each year of cardiovascular diseases, which is about $31 \%$ of all deaths worldwide. Coronary artery disease is the most common type of heart disease, killing over 370,000 people annually (Figure 4).

Headaches: A headache is a pain in the head or upper neck. They can be classified on the basis of their cause. They are primary and secondary. Primary headaches do not indicate any injury or medical condition while secondary headaches are associated with an injury or medical condition [9].

Primary headaches include migraines, cluster headaches and tension headaches. Secondary headaches are caused by medical conditions such as acute sinusitis, brain tumor, brain aneurysm and encephalitis.

Some primary headaches can also be triggered by certain lifestyle factors such as alcohol intake, especially red wine, smoking and sedentary lifestyle. Nearly 1 in 4 U.S. households includes someone with migraine. Amazingly, $12 \%$ of the population - including children - suffers from migraine. Migraine is the $3^{\text {rd }}$ most prevalent illness in the world. It is most common in between the age group of 18-44 (Figure 5).

Societal indicators of reductions in human energy expenditure and increases in sedentary behavior during the past several decades are particularly striking. In 1970, 2 in 10 working Americans were in jobs requiring only light activity (predominantly sitting at a desk), whereas 3 in 10 were in jobs requiring high-energy output (eg,

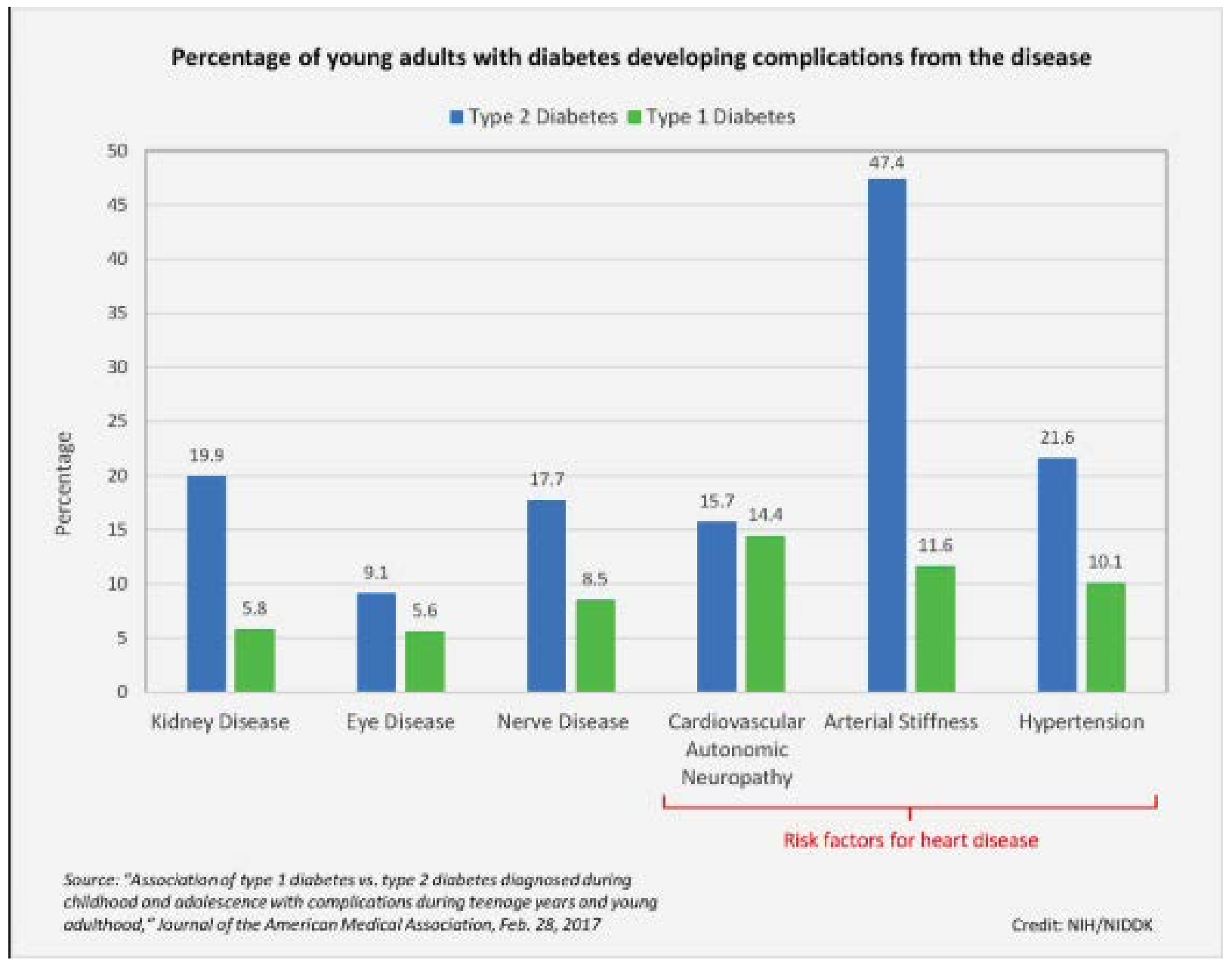

Figure 1: Percentage of young adults with diabetes developing complications from the disease. 


\section{Diabetes cases by age group}

A new analysis from UCLA finds that $55 \%$ of adults in California have either diabetes or pre-diabetes.

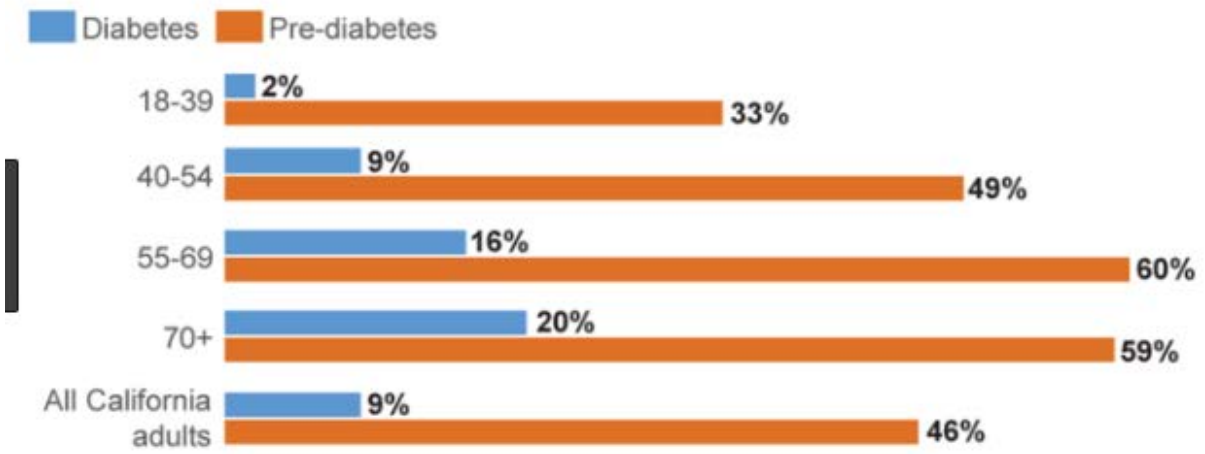

Figure 2: Diabetes cases by age group.

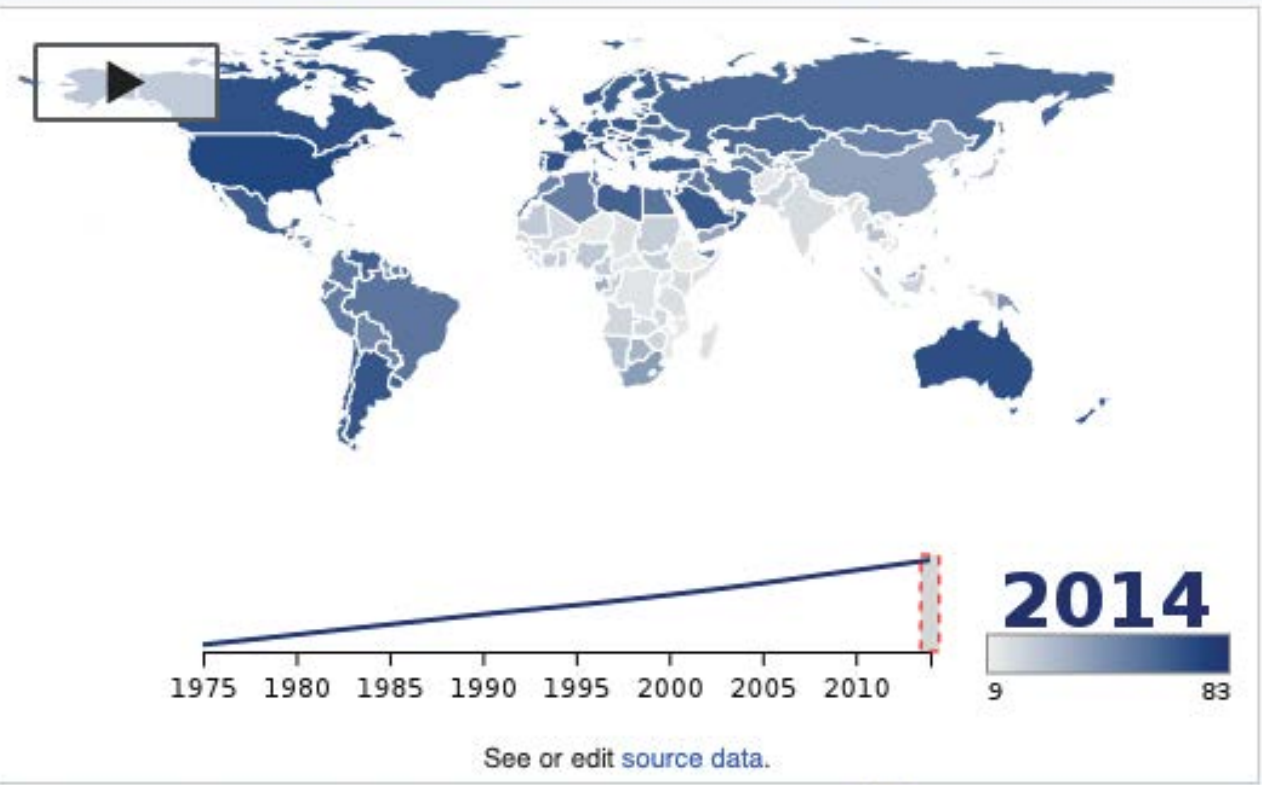

Percentage of the population either overweight or obese by year. ${ }^{[189]}$

Figure 3: Percentage of the population either overweight or obese by year.

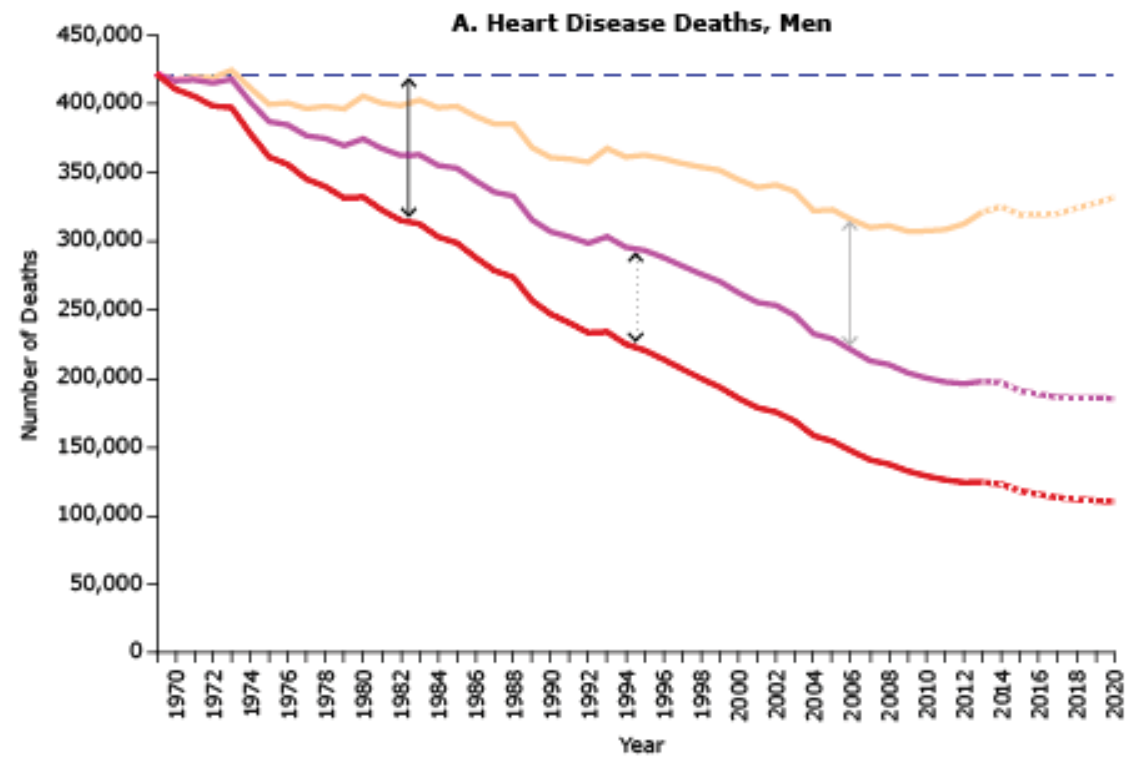

Figure 4: Heart disease deaths, Men. 
construction, manufacturing, farming). By 2000, more than 4 in 10 adults were in light-activity jobs, whereas 2 in 10 were in highactivity jobs. Moreover, during the past 20 years, total screen time (i.e., using computers, watching television, playing video games) has increased dramatically. In 2003, nearly 6 in 10 working adults used a computer on the job and more than 9 in 10 children used computers in school (kindergarten through grade 12). Between 1989 and 2009, the number of households with a computer and Internet access increased from $15 \%$ to $69 \%$. Other significant contributors to daily sitting time-watching television and driving personal vehicles-are at all-time highs, with estimates of nearly 4 hours and 1 hour, respectively [10].

\section{DIABETES}

According to an article in BMJ journals, a research conducted including men of different ethnicities concluded that the risk and prevalence of diabetes was directly correlated to sedentary lifestyles and lack of physical activity. Though leisure sedentary behaviors and television were significantly associated with the risk of diabetes, it was also influenced by race/ethnicity with most associations only being significant in non-Hispanic whites. Lower leisure behaviors were inversely associated with the incidence of type 2 diabetes. Several trials have shown that weight loss and physical activity reduce short term and long term incidence of type 2 diabetes $[11,12]$. It has been hypothesized that the main behavioral cause of insulin resistance is physical inactivity.

Past: A steep rise in the incidence of diabetes undoubtedly occurred in the later part of the 1900s. Regression analysis demonstrated that the incidence of diabetes doubled over a 30-year period from the 1950s, apparently peaking in the late 1970s. This rise is hypothesized to have been due to the increase in the number of desk jobs requiring low to almost no activity. The rise in incident diabetes type 2 is attributed to the unhealthy eating habits and drinking and smoking but the underlying behavioral cause is the lack of physical activity [13-15].

Current: In 2018, 23 million U.S. adults were diagnosed with diabetes. In 2016, the estimated prevalence of diagnosed type 1 and type 2 diabetes were $0.55 \%$ (corresponding to 1.3 million U.S. adults) and $8.6 \%$ (corresponding to 21.0 million U.S. adults), respectively. Type 1 and type 2 diabetes accounted for approximately $6 \%$ and $91 \%$ of all cases of diagnosed diabetes, respectively. The onset of the modern age in the 1950s has clearly shown a steep rise in the prevalence of diabetes in teens as well as adults, indicating a dependence of the disease on sedentary lifestyle as the onset of the 1950s came with the increase in the number of high stress jobs requiring low to virtually no activity [16-20].

\section{CARDIOVASCULAR DISEASES}

The most common cardiovascular disease associated with an inactive lifestyle is coronary artery disease and, various studies have shown that regular physical activity decreases the incidence of coronary artery disease significantly. The correlation between coronary artery disease and lack of physical activity makes it a preventable disease at the primary level. In developed countries, cardiovascular diseases are the number one cause of death, despite the fact that primary prevention is easily accessible. This may be correlated to the high number of jobs requiring low or no physical activity. Regular physical fitness helps prevent diabetes, maintain weight loss and has a positive effect on hypertension, which are all independent factors of cardiovascular diseases $[21,22]$.

Past: Approximately 40 years ago, Morris et al reported that middle-aged men who perform vigorous physical activity in their leisure time on at least 2 days/week have a one third lower likelihood of developing coronary artery disease than their inactive peers. Various studies have also shown a direct relation between cardiovascular diseases and physical inactivity. It is notable that there was a sharp increase in the incidence of cardiovascular diseases from 1900 to 1960 after which, the incidence rate showed a steady decline. This has been hypothesized to be due to the advanced medical techniques developed and available. Total CVD mortality declined by $68 \%$ between 1980 and 2013 in the UK $[23,24]$. Similar decreases were seen for coronary heart disease and stroke (Figure 6).

Current: It is seen that though mortality rates due to cardiovascular diseases have decreased significantly, the number of hospital admissions due to cardiovascular diseases has increased. This is attributed to better available treatments and medical research with time but the number of hospital admissions has been on a steady rise, which may also be attributed to the increasingly sedentary lifestyle with stressful jobs requiring the minimum amount of

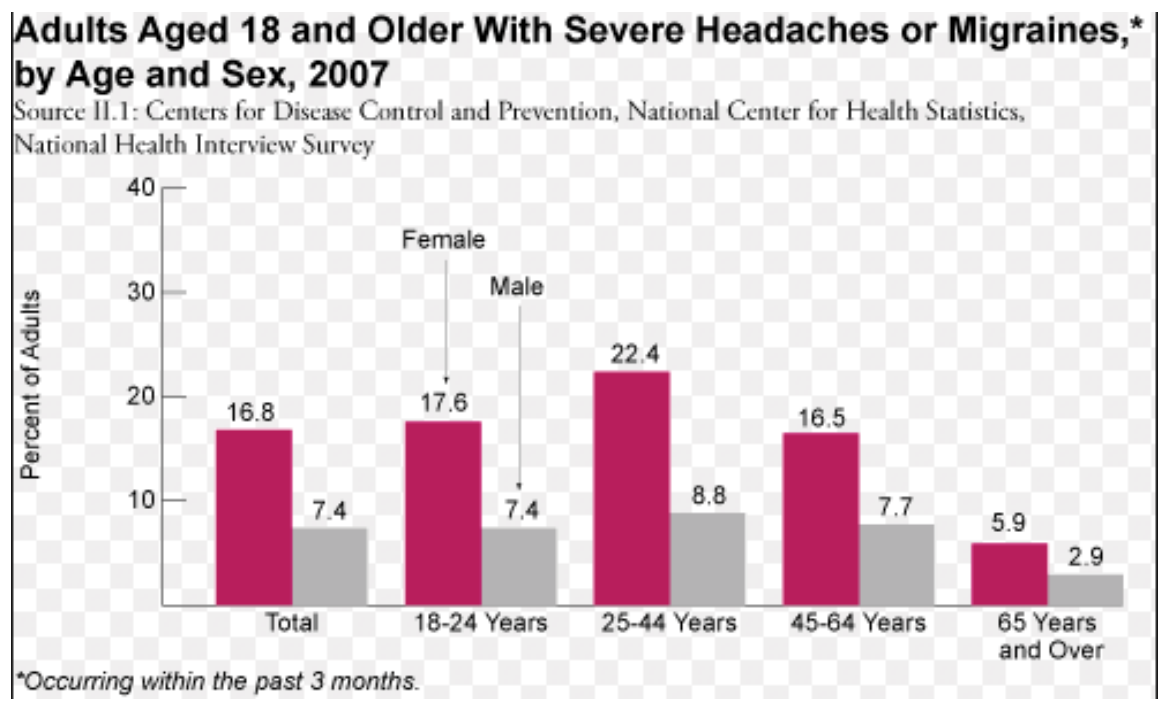

Figure 5: Adults aged 18 and older with severe headaches or migraines, by age any sex, 2007. 
activity and the populations sitting in front of their televisions at home after work instead of having some physical activity [25]. It is notable that even though coronary artery disease is preventable at the primary stage, its incidence rate is still increasing (Figure 7).

\section{OBESITY}

Obesity is the underlying cause of most diseases like coronary artery disease and diabetes. According to WHO, the incidence of obesity has nearly tripled since 1975 most of the world's population lives in countries where overweight and obesity kills more people than underweight? There is a direct correlation between obesity and physical inactivity, which is why it is a preventable disease. In 2016, 1.9 billion adults worldwide were overweight and 650 million were obese. WHO also attributes obesity to physical inactivity due to the increasingly sedentary lifestyles, changing modes of transportation and urbanization [26].

Past: The prevalence of obesity has seen a steady increase since the mid-1950s to the 21 st century century. The level of physical activity has also shown a steep decline in this period. Collectively, the number of overweight and obese people worldwide has increased from 857 million in 1980 to 2.1 billion in 2013 .

Current: More than 2 billion people worldwide are overweight/ obese. Though the rate of increase in obesity has not showing any significant change in some developed countries for the past few years, it is not certain whether the obesity epidemic has peaked or the rates are starting to decrease due to awareness and preventative methods which have been seen in the past few years with the introduction of "active desks", exercise balls in place of office chairs and other means where employees can work while taking care of their physical health. This also shows more productivity and better morale in employees [27] (Figure 8).

\section{HEADACHES}

The cause of headaches (without an underlying disease) is rooted in the stress and lifestyle and other such common factors in one's life. With the changing lifestyles and increasing urbanization, came the steady and steep rise in incidence of headaches. Studies show that headaches can have a direct or indirect correlation to the lifestyle of a person. With the increase in high stress jobs with low physical activity since the mid-20th century, showing a correlation with the increase in a comfortable/sedentary lifestyle [28-32].

Past: Headaches have always been prevalent but a sudden increase was seen in the 1900s, supposedly owing to the increase in stress and the presence of screens all around with the development of technology which has shown to be a cause of headaches in several studies. The use of these devices with digital screens is also indirectly related to a sedentary lifestyle as people have a tendency to use these devices while sitting or lying down.

Current: Primary headaches are a common problem in the general population and 1 of the most common complaints in neurology clinics. Migraine has a prevalence of approximately $14 \%$ worldwide and contributes to around $40 \%$ to $50 \%$ of the overall headache burden. Tension-type headache is the most prevalent primary headache in the population and contributes to $60 \%$ of the total headache burden. The number of cases of headaches has had a continuous increase since the 2000s with increase in usage of smartphones and laptops and physical inactivity [33].

\section{DATA ANALYSIS AND INTERPRETATION}

Sample size: 57 , predominantly females $(63.1 \%)$;

Predominantly $16-17$ years old $(63.1 \%)$;

Age Group: 16-18 years age group participated majorly [34-37].

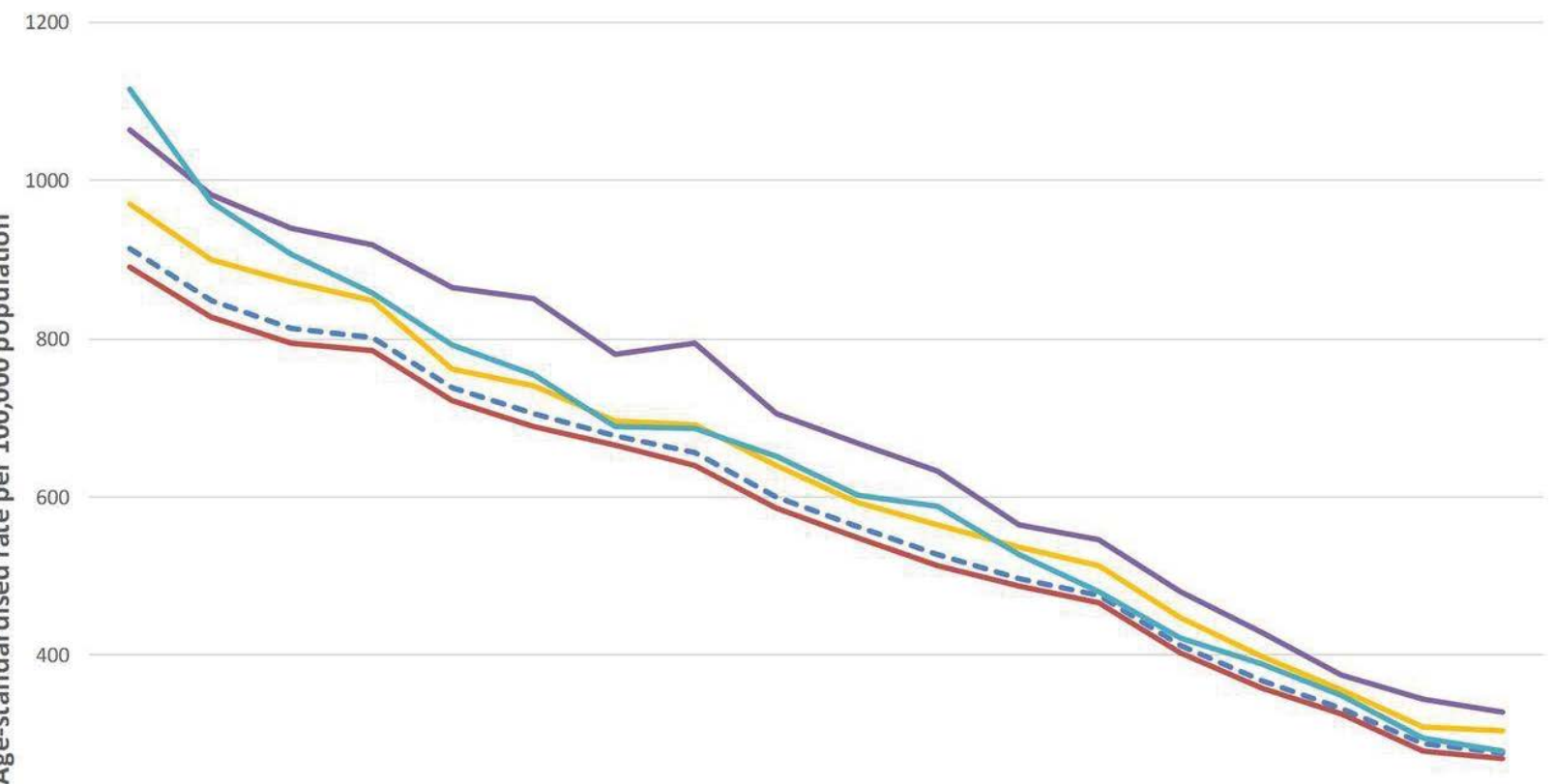

200

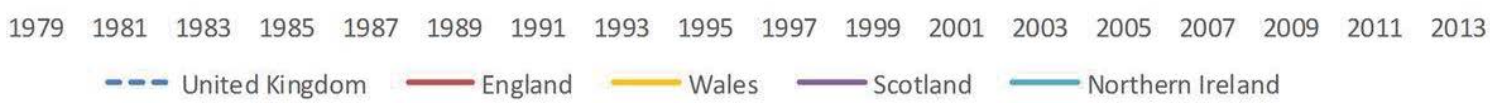

Figure 6: Age standardized rate per 100,000 populations. 


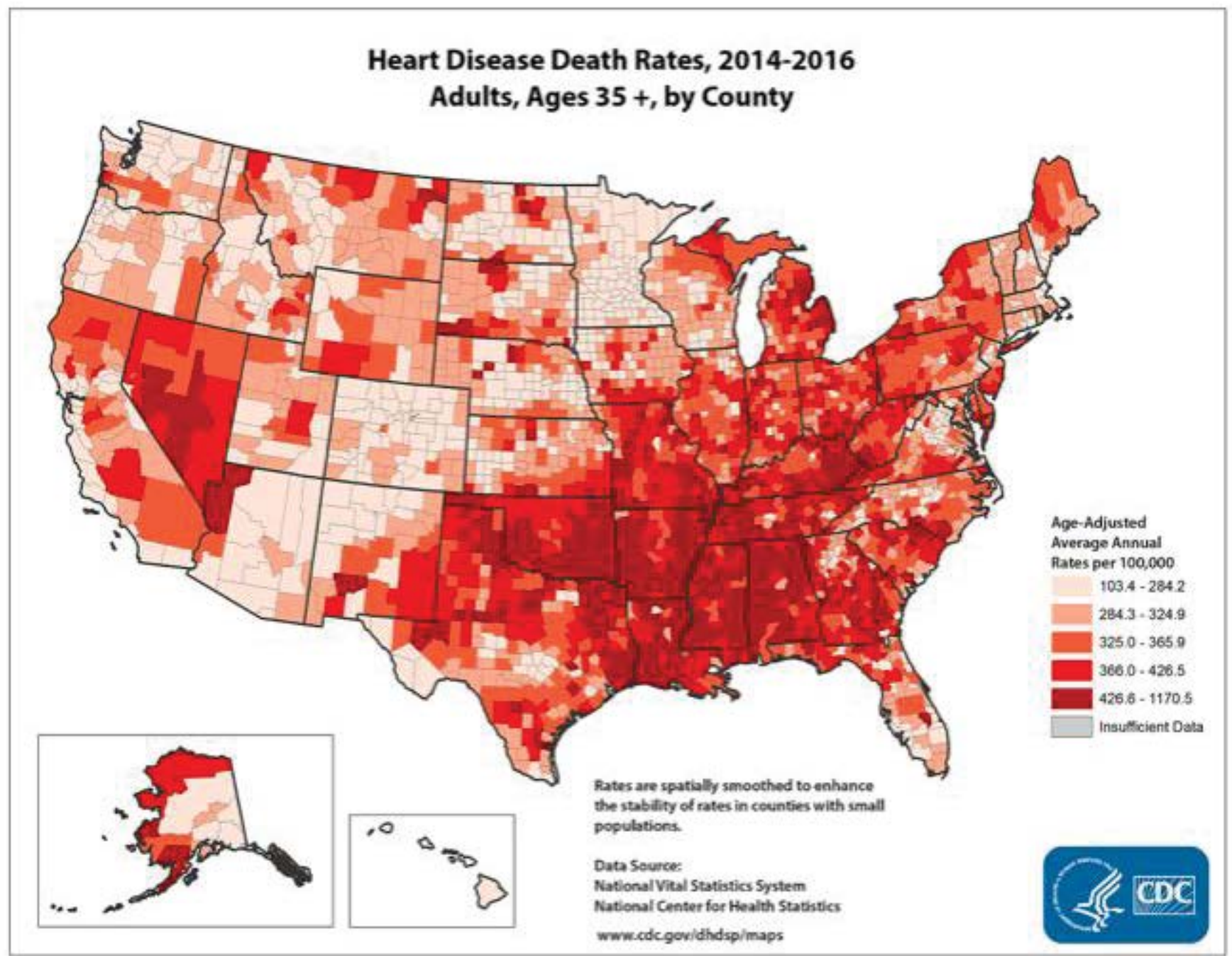

Figure 7: Heart disease death rates, 2014-2016.

\section{Trends in adult overweight, obesity, and extreme obesity among men and women aged 20-74: United States, 1960-1962 through 2013-2014}

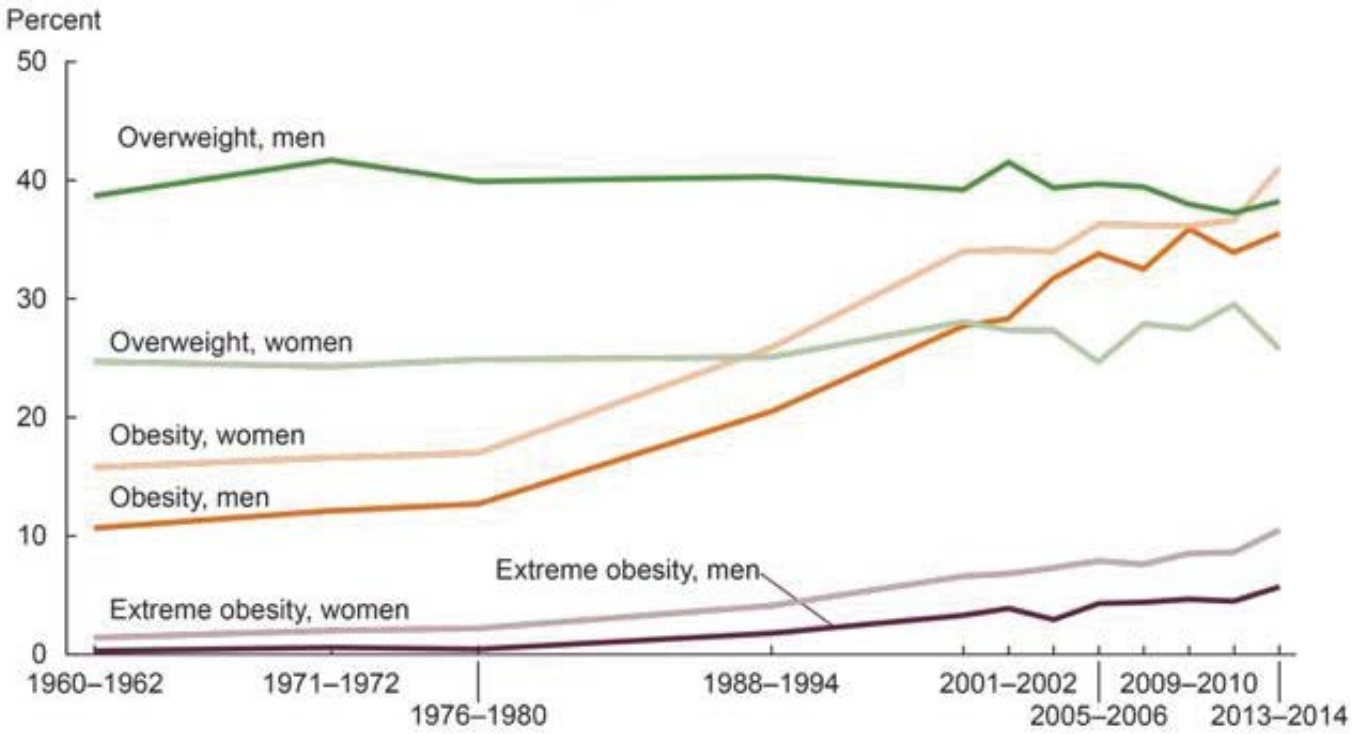

NOTES: Age-adjusted by the direct method to the year 2000 U.S. Census Bureau estimates using age groups 20-39, 40-59, and 60-74. Overweight is body mass index (BMI) of $25 \mathrm{~kg} / \mathrm{m}^{2}$ or greater but less than $30 \mathrm{~kg} / \mathrm{m}^{2}$; obesity is BMI greater than or equal to 30 ; and extreme obesity is BMI greater than or equal to 40 . Pregnant females were excluded from the analysis.

greater than or equal to 40, Pregnant females were excluded from the analysis.
SOURCES: NCHS, National Health Examination Survey and National Health and Nutrition Examination Surveys.

Figure 8: Trends in adults overweight, obesity, and extreme obesity among men and women aged 20-74: US, 1960-1962 through 2013-2014. 
Survey was done using an online platform, mainly targeting the high school and college going population of Chandigarh, India.

Qualifications: Currently studying qualifications (Figure 9).

How many hours do you spend on video games on weekly basis?

According to the data analysis, it was interpreted that $75.4 \%$ of the teenagers spend 0-1 hours on playing video games (Figure 10).

How many hours a week do you spend on TV?

The data interpretation shows that $22.8 \%$ of teenagers spend $1-2$ hours $\mathrm{n}$ watching TV in a week whereas $12.3 \%$ of the teenagers spend more than 5 hours in a week (Figure 11).

How many hours a week do you watch Netflix and the other online streaming services?

This showed that approximately $45 \%$ of people spent an unhealthy amount of time on online streaming services when they could engage in some sort of physical activity (Figure 12).

How much exercise do you get weekly?

This showed that about $55 \%$ of those surveyed did not achieve the minimum weekly exercise quota recommended by the Department of Health and Human Services (Figure 13).

Do you get short of breath while walking even for 10-15 minutes?

This showed that $8.8 \%$ surveyed got short of breath after walking for 10-15 minutes while 15.8\% were not sure (Figure 14).

Do you get heartburn, pain, tightness or any discomfort in the chest?

$17.5 \%$ of the population surveyed reported incidences of heartburn and pain or discomfort in the chest, indicating early effects of a sedentary lifestyle on the cardiovascular system Figure 15.

Do you get frequent headaches while watching screens?

The data interpretation shows that $59.6 \%$ of the total sample size does not get frequent headaches while watching screen (Figure 16).

Do you get easily fatigues?

$15.8 \%$ reported feeling easily fatigued while performing basic tasks while working on the daily routine tasks (Figure 17).

Do you have an excessive thirst/ hunger?

$33.3 \%$ of the total sample of teenagers felt excessive thirst/ hunger whereas $40.4 \%$ of the total strength does not feel the urge (Figure 18).

How irritable are you?

$43.9 \%$ of the total strength gets irritated quite often whereas $14 \%$ of these gets irritated excessively and leads to short temperament (Figure 19).

How often do you eat junk food?

The population surveyed predominantly ate junk food more often than once a week (about 70\%) (Figure 20).

\section{CONCLUSION}

This shows that the rate of diseases like obesity, cardiovascular diseases and diabetes type- 2 has increased substantially since the 1950s, which may be attributed to the increased availability, affordability and range of different machines for different purposes which has made life easier and at the same time brought human

\section{Qualifications/currently studying}

57 responses

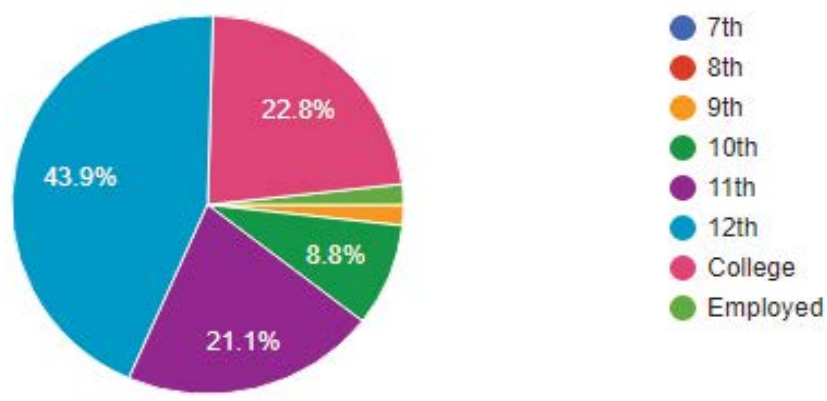

Figure 9: Qualifications.

\section{How many hours do you spend on video games on a weekly basis}
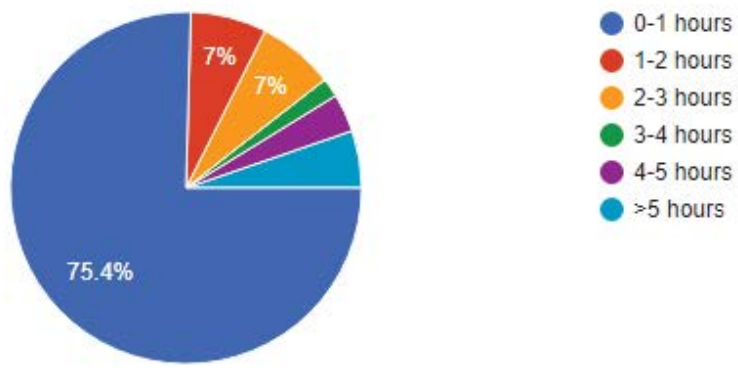

Figure 10: How many hours do you spend on video games on weekly basis. 
how many hours a week do you spend on TV

57 responses

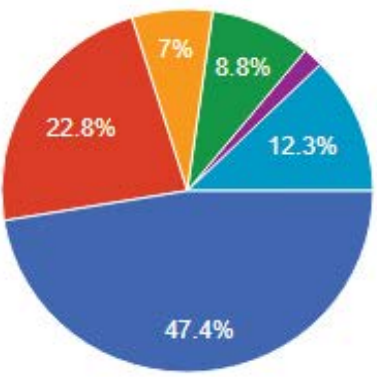

Figure 11: How many hours a week do you spend on TV.

How many hours a week do you watch Netflix and other online streaming services

57 responses
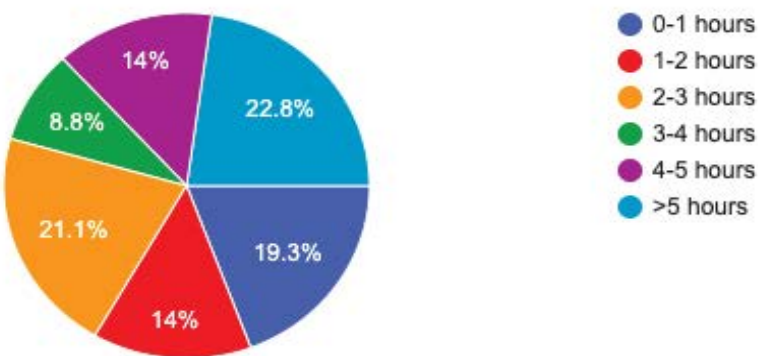

Figure 12: How many hours a week do you watch Netflix and the other online streaming services.

\section{How much exercise do you get weekly?}

57 responses

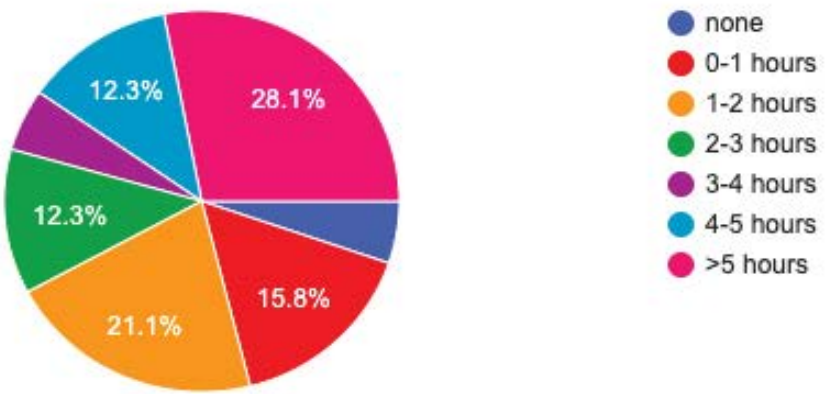

Figure 13: How much exercise do you get weekly?

\section{Do you get short of breath while walking even for 10-15 minutes}

\section{7 responses}
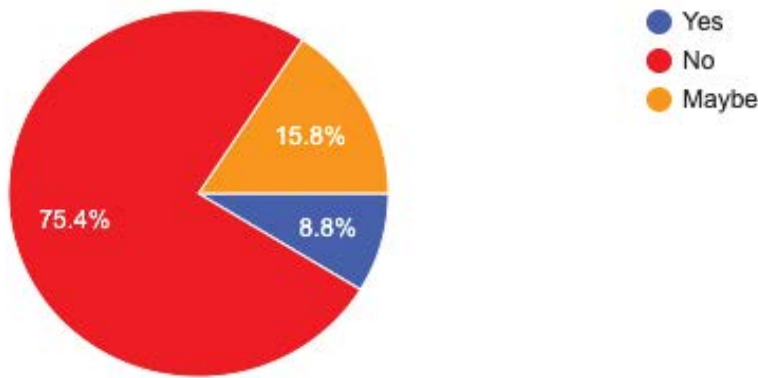

Figure 14: Do you get short of breath while walking even for 10-15 minutes? 


\section{Do you get heartburn, pain, tightness or any discomfort in the chest}

57 responses

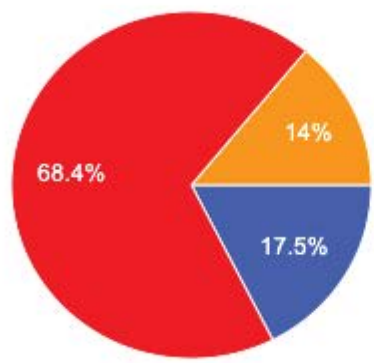

$$
\begin{aligned}
& \text { Yes } \\
& \text { No } \\
& \text { Maybe }
\end{aligned}
$$

Figure 15: Do you get heartburn, pain, tightness or any discomfort in the chest?

\section{Do you get frequent headaches while watching screens}

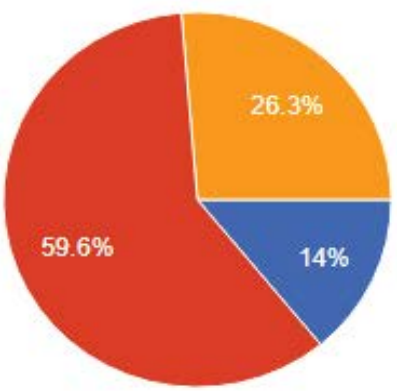

Figure 16: Do you get frequent headaches while watching screens.

\section{Do you get easily fatigued}

57 responses

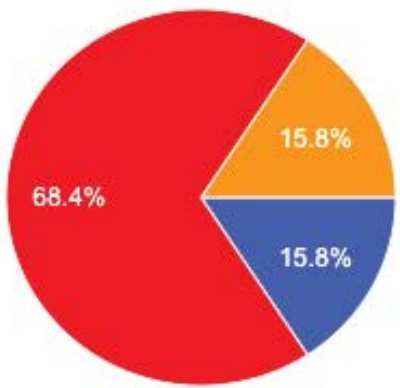

Figure 17: Do you get easily fatigues.

\section{Do you have excessive thirst/hunger}

\section{7 responses}

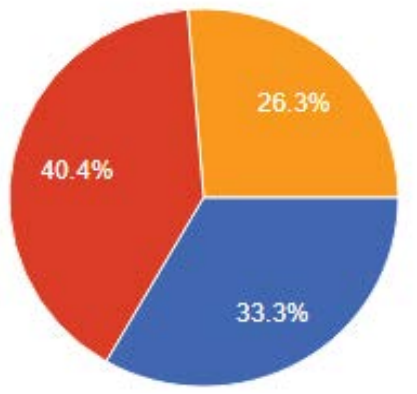




\title{
How irritable are you
}

57 responses

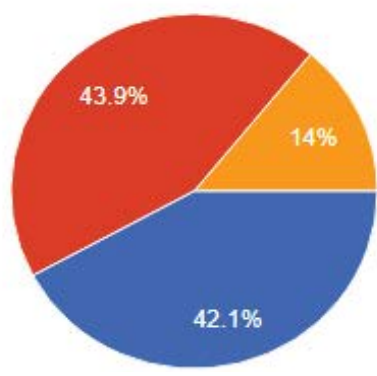

Figure 19: How irritable are you.

\section{How often do you eat junk food}

\author{
57 responses
}

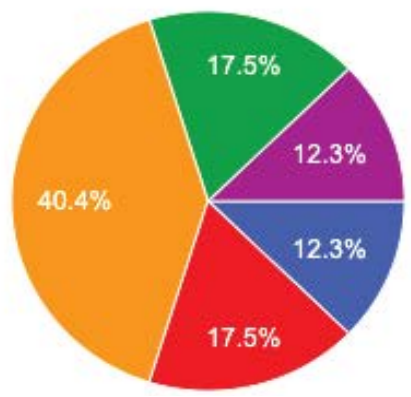

Monthly

Every 15 days or so

Weekly

More than thrice a week

Almost daily

Figure 20: How often do you eat junk food.

energy expenditure to a relative stand still. The availability of modern means of transport and increasing urbanization has been directly correlated to the prevalence of these diseases/disorders. It is surprising to see that even though it is preventable at the primary stage and innumerable efforts have been made to raise awareness of the disorders accompanied by sedentary behavior, it still exists as one of the largest reasons for deaths worldwide.

Physical inactivity has also been linked with depression, anxiety and other mental disorders. Assumptions have been made that physical inactivity causes more deaths annually than smoking. Physical inactivity is the root of many other potentially fatal disorders.

\section{REFERENCES}

1. Hornshaw M. More than 650 million adults are obese, Obesity is killing us: Obesity profoundly perturbs the metabolome. 2018

2. WHO. 10 facts on obesity. 2017.

3. Ssewanyana D, Abubakar A, Anneloes B, Patrick NM, Charles RN. Perspectives on Underlying Factors for Unhealthy Diet and Sedentary Lifestyle of Adolescents at a Kenyan Coastal Setting. 2018.

4. Manuela FR, Luciano MF, Raquel MS, Ameliane, Paula R, Clara MS. Urban versus rural lifestyle in adolescents: associations between environment, physical activity levels and sedentary behavior. Einstein. 2016;14(4):461-467.

5. https://www.hopkinsmedicine.org/health/conditions-and-diseases/ risks-of-physical-inactivity

6. Health Risks of a Sedentary Lifestyle. 2017.

7. WHO. Physical inactivity a leading cause of disease and disability, warns WHO. 2002.
8. NCD Riskfactor. Worldwide trends in diabetes since 1980: a pooled analysis of 751 population-based studies with $4 \llbracket 4$ million participants. 2016;387(10027):1513-1530.

9. https://medlineplus.gov/diabetes.html

10. https://en.wikipedia.org/wiki/Obesity

11. Coronary artery disease. Patient Care \& Health Information.

12. Statistics about Diabetes. 2015.

13. CDC. Heart Disease Facts.

14. WHO. Obesity and overweight. 2018.

15. https://migraineresearchfoundation.org/about-migraine/migrainefacts/

16. American Diabetes Association. 2017.

17. Edwin AMG. The Rise of Childhood Type 1 Diabetes in the 20th Century. 2002;51(12):3353-3361.

18. Kai McKB, Catherine CC, Sarah EL, Sharon HS, Andy M, Linda SG, et al. Prevalence of Diagnosed Diabetes in Adults by Diabetes Type - United States, 2016. MMWR Morb Mortal Wkly Rep. 2018, 67(12):359-361

19. Sara P. How Much Have Obesity Rates Risen Since 1950.

20. https://www.who.int/health-topics/cardiovasculardiseases/\#tab=tab_1

21. Ephraim BW, Felix W, Axel L. Physical Activity in the Prevention and Treatment of Coronary Artery Disease. J Am Heart Assoc. 2018; 7(4):pp:e007725

22. Prachi B, Kremlin W, Elizabeth W, Nick T. Trends in the epidemiology of cardiovascular disease in the UK. BMJ. 2016:102(24). 
23. Uemura K, Pisa Z. Trends in cardiovascular disease mortality in industrialized countries since 1950. World Health Stat Q. 1988;41(3):155-178

24. https://www.cdc.gov/dhdsp/data_statistics/fact_sheets/fs_heart_ disease.htm

25. Heart Disease and Stroke Statistics-2019 At-a-Glance. American Heart Association.

26. NIH. Overweight \& Obesity Statistics.

27. Study suggests worsening trends in headache management. 2015.

28. Nacharin P, Chaichana N, Anan S, Shuu JW. Epidemiology of headache. 2001.

29. Neville O, Phillip BS, Genevieve NH, David WD, Charles EM. Sedentary Behavior: Emerging Evidence for a New Health Risk. 2010; 85(12):1138-1141.
30. Joshua JJ, Justin BET, Sherita HG, Haiying C, Nancy S, Mercedes RC, et al. BMJ. 2015;4(1)

31. https://www.health.ny.gov/diseases/chronic/cvd.htm

32. https://www.medicalnewstoday.com/articles/277450.php

33. Chooi YC, Ding C, Magkos F. The epidemiology of obesity. Metabolism. 2019;92:6-10.

34. Hannah KW, Robert NA, Sallyann MCK, Ashwini S, Trevor DT, Yuling $\mathrm{H}$, et al. Heart Disease and Cancer Deaths - Trends and Projections in the United States, 1969-2020. CDC. 2016; 13.

35. https://mchb.hrsa.gov/whusa09/hstat/hi/pages/230shm.html

36. https://www.cdc.gov/dhdsp/maps/atlas/index.htm

37. https://docs.google.com/forms/ d/1RZOKTWUDG1wKjDEggMnLm__Rb8BRC5lj_b-4lyDhKPk/ edit\#responses 